\title{
A versatile building block: the structures and functions of negative-sense single-stranded RNA virus nucleocapsid proteins
}

\author{
Yuna Sun ${ }^{1,2}$, Yu Guo ${ }^{3,4}$, Zhiyong Lou ${ }^{2}$ \\ ${ }^{1}$ National Laboratory of Macromolecules, Institute of Biophysics, Chinese Academy of Science, Beijing 100101, China \\ 2 Laboratory of Structural Biology and MOE Laboratory of Protein Science, School of Medicine and Life Sciences, Tsinghua \\ University, Beijing 100084, China \\ ${ }^{3}$ High-throughput Molecular Drug Discovery Center, Tianjin Joint Academy of Biomedicine and Technology, Tianjin 300457, \\ China \\ ${ }^{4}$ College of Pharmacy and State Key Laboratory of Medicinal Chemical Biology, Nankai University, Tianjin 300071, China \\ $\bowtie$ Correspondence: louzy@xtal.tsinghua.edu.cn
}

Received August 22, 2012 Accepted September 3, 2012

\begin{abstract}
Nucleocapsid protein (NPs) of negative-sense singlestranded RNA (-ssRNA) viruses function in different stages of viral replication, transcription, and maturation. Structural investigations show that -ssRNA viruses that encode NPs preliminarily serve as structural building blocks that encapsidate and protect the viral genomic RNA and mediate the interaction between genomic RNA and RNA-dependent RNA polymerase. However, recent structural results have revealed other biological functions of -ssRNA viruses that extend our understanding of the versatile roles of virally encoded NPs.
\end{abstract}

KEYWORDS -ssRNA virus, nucleocapsid protein, crystal structure, function

\section{INTRODUCTION}

Negative-sense single-stranded RNA (-ssRNA) viruses are some of the most important pathogens to cause severe infectious diseases among humans, animals, and plants. Currently, two orders and seven families, together with a number of unassigned species and genera, are recognized in the -ssRNA group (Lansing et al., 1998; Ruigrok et al., 2011) (Table 1). A -ssRNA virus is identified by its genomic RNA, which comprises one or several antisense RNA segment(s) (Xue et al., 2010). Once the -ssRNA virus intrudes host cells, the genome is transcribed into the mRNA by viral RNA-dep- endent RNA polymerase (RdRp) such that the genomic RNA and $R d R p$ are included in the virions of the virus. During this process, RdRp does not directly interact with genomic RNA in the virion, and a virally encoded nucleocapsid protein (NP) is necessary to mediate their interaction.

NPs also encapsidate and package genomic RNA to prevent it from degradation by exogenous nucleases or innate immune systems in the host cell. As a result, the viral RdRp, genomic RNA, and NP form a stable complex (RNP) that accomplishes viral replication and transcription throughout the viral life cycle (Kranzusch and Whelan, 2012).

A number of virally encoded accessory proteins or host cell factors are involved in the formation of the virus replication complex, and understanding the detailed three-dimensional structure and precise molecular mechanism of the assembly of RNP from -ssRNA virus, as well as the biological functions of viral NP, is of great importance.

Structural information on several -ssRNA viruses that encode NPs throughout a wide range of virus families has been reported, including influenza virus (Orthomyxovidae) (Ye et al., 2006; $\mathrm{Ng}$ et al., 2008), rabies virus (Rhabdoviridae) (Albertini et al., 2006), vesicular stomatitis virus (VSV) (Rhabdoviridae) (Green et al., 2006), borna disease virus (BDV) (Bornaviridae) (Rudolph et al., 2003), Arenavirus (Hastie et al., 2011a; Hastie et al., 2011b; Qi et al., 2011), and Bunyavirus (Raymond et al., 2010; Ferron et al., 2011; Guo et al., 2012), among others. Many studies have presented how NPs encapsidate genomic RNA and oligomerize to form highly ordered structures. Interestingly, recent results have 
Table 1 Negative ssRNA viruses nucleoproteins discussed in this review

\begin{tabular}{|c|c|c|c|c|}
\hline Order & Family & Subfamily & Typical species & PDB codes \\
\hline \multirow{6}{*}{ 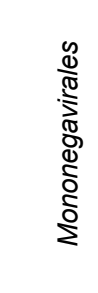 } & \multicolumn{2}{|l|}{ Phabdoviridae } & Rabies virus & 2GTT \\
\hline & \multirow{3}{*}{ Paramyxoviridae } & \multirow{5}{*}{$\begin{array}{l}\text { Paramyxovirinae } \\
\text { Pneumovirinae }\end{array}$} & VSV & $2 \mathrm{GIC}$ \\
\hline & & & Measles virus, Sendai virus & $\mathrm{N} / \mathrm{A}$ \\
\hline & & & RSV & 2WJ8 \\
\hline & \multirow{2}{*}{$\begin{array}{l}\text { Bornaviridae } \\
\text { Fornaviridae }\end{array}$} & & BDV & 1N93, 1PP1 \\
\hline & & & Marburg Virus, Ebola Virus & $\mathrm{N} / \mathrm{A}$ \\
\hline \multirow{6}{*}{ 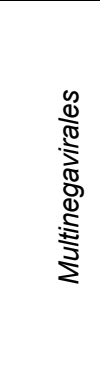 } & \multirow{2}{*}{\multicolumn{2}{|c|}{ Arenaviridae }} & LASV & $3 \mathrm{M} \times 2$ \\
\hline & & & & $3 Q 78$ \\
\hline & & & & $3 T 5 Q$ \\
\hline & \multirow[t]{2}{*}{ Bunyaviridae } & & RVFV & $\begin{array}{l}\text { 3LYE } \\
30 U 9\end{array}$ \\
\hline & & & CCHFV & $\begin{array}{l}3 \mathrm{UI} \\
4 \mathrm{AKL} \\
4 \mathrm{AQF}, 4 \mathrm{AQG}\end{array}$ \\
\hline & \multicolumn{2}{|l|}{ Orthomyxoviridae } & Influenza virus & $\begin{array}{l}\text { 2IQH 2Q06 } \\
\text { 3TJ0 }\end{array}$ \\
\hline
\end{tabular}

Abbreviations: VSV: Vesicular stomatitis virus; RSV: Respiratory syncytial virus; BDV: Borna disease virus; LASV: Lassa fever virus; RVFV: Rift Valley fever virus; CCHFV: Crimean Congo Hemorrhagic Fever Virus

revealed the unexpected biological (enzymatic) functions of these virally encoded NPs. This review will summarize the current knowledge of the biological functions, as well as the interesting structural features of -ssRNA virus NPs.

\section{NP FUNCTIONS IN RNA ENCAPSIDATION}

The first detailed elucidation of the structure and molecular mechanism of how NP encapsidates RNA was demonstrated by the crystal structures of the NP-RNA complexes of Rhabdoviridae and Paramyxoviridae members. VSV and rabie virus, which belong to the Rhabdoviridae family, cause severe human diseases ( $\mathrm{Ge}$ et al., 2010). Although the biologies of VSV and rabie virus are different, their replication and transcription share similarities (Albertini et al., 2008). The genomic RNA of VSV and rabie virus is encapsidated by their NPs, and this process requires a viral L-P polymerase complex for transcription and replication. $\mathrm{P}$ protein (phosphoprotein) is a key accessory factor for the regulation of polymerase activity (Green and Luo, 2009) (Fig. 1). In this process, the NPs bind the viral genome with a stoichiometry of a few nucleotides per NP-protomer in both VSV and rabie virus to form the template for viral polymerases, instead of genomic RNA alone (Thomas et al., 1985; Emerson and Schubert, 1987; Iseni et al., 1998). P protein subsequently binds with both $L$ and the NP-RNA complex and mediates their interaction to start the polymerase reaction. Recombinant NPs from VSV and rabie virus form long helical structures or inhomogeneous ring-shaped structures with 9-15 NP-protomers per ring, together with bound cellular RNA (Albertini et al., 2008). NPs from VSV (PDB code: $2 \mathrm{GIC}$ ) and rabie virus (PDB code: 2GTT) present similar patterns of molecular folding, including an $\mathrm{N}$-lobe and a $\mathrm{C}$-lobe (Fig. $2 \mathrm{~A}$ and $2 \mathrm{~B}$ ). A two- $\beta$-stranded arm in the $\mathrm{N}$-terminus of the $\mathrm{N}$-lobe and an extended loop structure within the $\mathrm{C}$-lobe are responsible for the formation of the ring-shaped structure by interacting with two adjacent NP-protomers. RNA binding grooves are located at the inter-

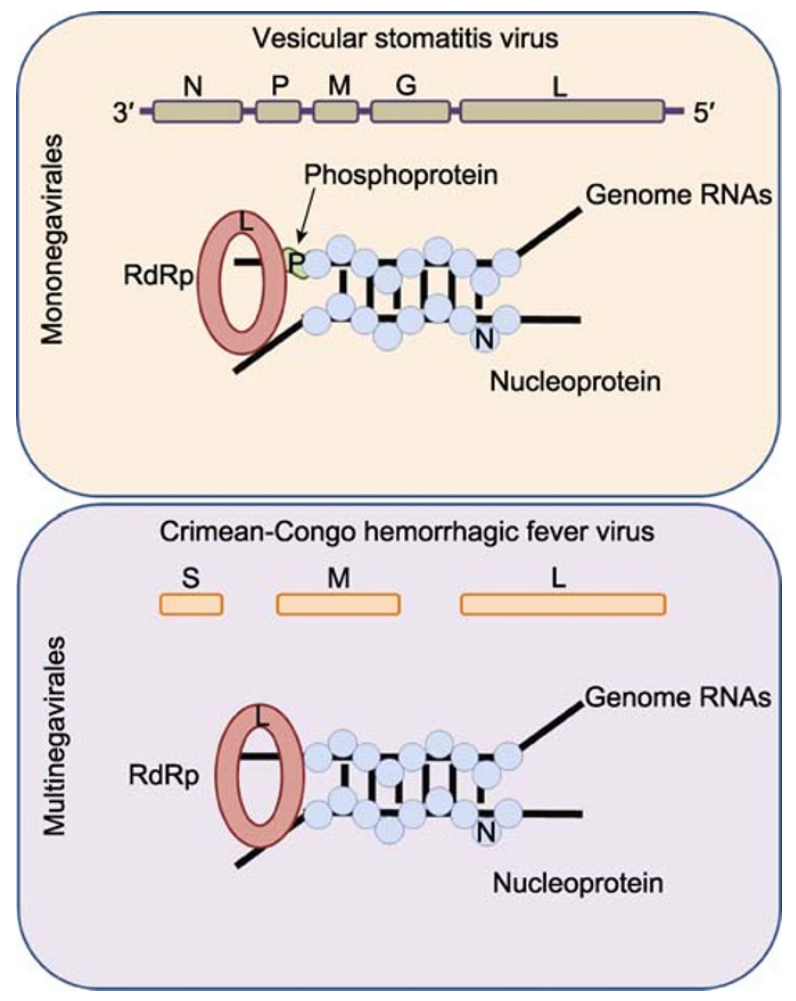

Figure 1. A schematic representation of virus replication complex. 
A
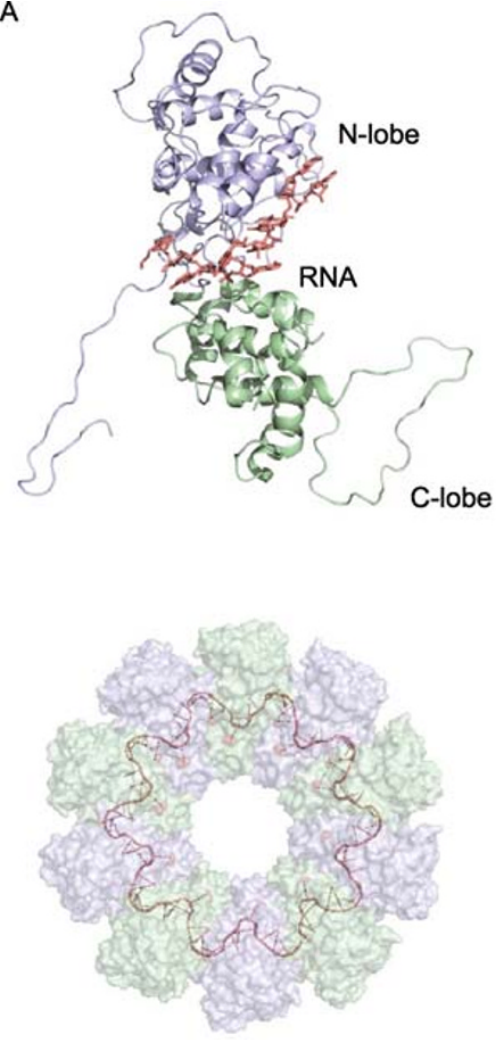

VSV NP-RNA
B
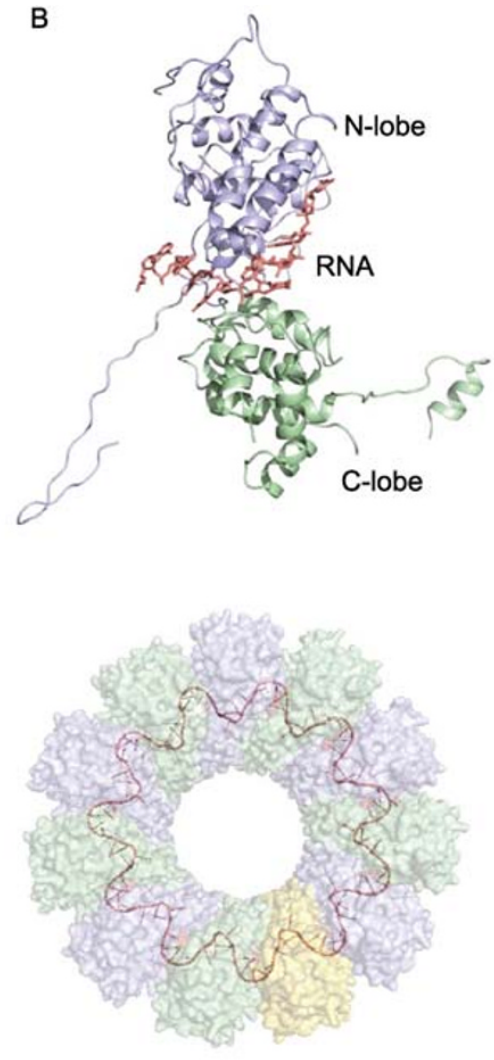

Rabie virus NP-RNA
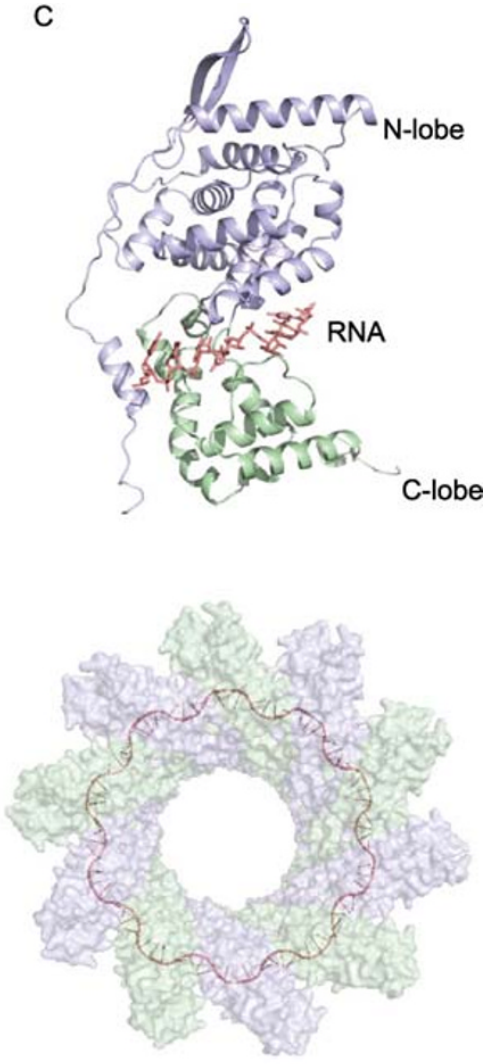

RSV NP-RNA

Figure 2. Comparison of nucleocapsid proteins of VSV, rabie virus and RSV. Crystal structures of NP-RNA complex from VSV (PDB code: $2 \mathrm{GIC}$ ) (A), rabie virus (PDB code: 2GTT) (B) and RSV (PDB code: 2 WJ8) (C) are aligned and displayed in the same orientation. The monomeric NP-RNA complexes are shown at upper panels, and the oligomeric ring-shaped complexes are presented at bottom panels. For the monomeric structures, the N-lobe and C-lobe are colored with blue and green, respectively, and the bound RNA molecules are shown as red sticks. Since there are 11 NP-protomers in one NP-RNA ring-shaped complex of rabie virus, one additional protomer is covered by yellow surface, while the other protomers are covered with blue and green surface.

faces of N- and C-lobe, and each NP protomer comes into contact with nine nucleotides with no sequence specificity. NPs from VSV and rabie virus could strongly bind and protect the bound RNA. Only extremely extensive treatment with exogenous nuclease can remove the bound RNA from the ring-shaped structure (Green et al., 2011). The stable NP-RNA complex protects the viral genome from host immune recognition or nuclease degradation. Although the structures of VSV and rabie virus NP-protomers are similar, the crystal structure of the VSV NP ring contains 10 protomers whereas 11 NP-protomers are in one rabie virus ring-shaped NP-RNA complex. The contents of NP-protomer in the ring-shaped structure are not strictly homogenous so this difference may only represent a crystallographic feature. In other words, the VSV NP-RNA complex containing 10 NP-protomers and the rabie virus NP-RNA complex containing 11 NP-protomers could give sufficient diffraction quality for structure determination.

A similar ring-shaped NP-RNA complex is observed in respiratory syncytial virus (RSV) (Tawar et al., 2009) (Fig. 2C). RSV belongs to the Paramyxoviridae family of the order Mononegavirales, which also includes the Rhabdoviridae, Bornaviridae, and Filoviridae families. Consistent with their viral homology, RSV NP presents a monomeric architecture similar to that of VSV and rabie virus and includes an N-lobe and a C-lobe to clamp the RNA binding site to its interface (PDB code: 2WJ8). However, several structural variations distinguish it from the VSV and rabie virus NP-RNA complex. First, RSV NPs contain only seven nucleotides while nine may be found in VSV and rabie virus NP-protomers. The most significant difference between them is the position of bound RNA. Although the RNA binding grooves of VSV/rabie virus and RSV NPs are located at the interface of the N-lobe and the C-lobe, the lateral $\mathrm{N}$ comes in contact with the VSV and rabie virus NP-RNA complex such that the curvature is opposite that of the RSV ring (Fig. 2, bottom panels). This position results in an inside-out nucleocapsid ring, with the RNA inside and the $\mathrm{N}$ molecule oriented outside-in (Tawar et 
al., 2009).

The variation in the highly ordered architecture of the NP-RNA complex also appears in other -ssRNA virus. Among these viral NPs, the NP from influenza virus shows the most variable organization. Like other -ssRNA viruses, influenza virus generates an RNP formed by genomic RNA, a nucleocapsid, and the PA-PB1-PB2 polymerase complex (Yuan et al., 2009; Zhao et al., 2009) for viral replication/ transcription. In the influenza virus RNP, one PA-PB1-PB2 polymerase complex attaches to the ring-shaped NP-RNA complex and thus functions in viral life cycle (Area et al., 2004). Research on the structural details of influenza virus achieved a breakthrough when two independent groups reported the crystal structure of RNA-free NP from influenza A virus (IAV) (PDB codes: 2IQH and 2Q06) (Ye et al., 2006; $\mathrm{Ng}$ et al., 2008). These two structures showed the crescent-shaped IAV NP with three distinct portions, called the head, body, and tail (Fig. 3 upper panels).

Although the sequence and detailed structure of IAV NPs

A
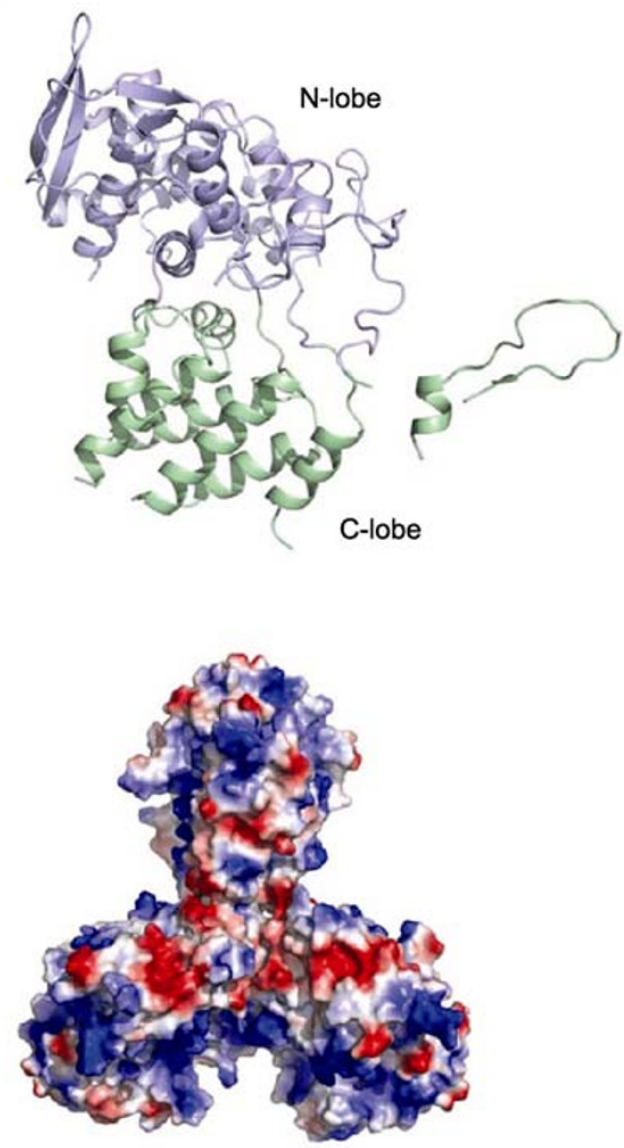

Influenza A show few similarities with those of VSV and rabie virus NPs, the overall architecture of the IAV NP molecule presents a deep, positively charged RNA binding groove clamped by the head and the body parts with the tail loop mediating the oligomerization of IAV NPs through interactions with neighboring molecules. These structural characteristics classified IAV NPs with the similar features identified by VSV and rabie virus NPs. However, the highly ordered structure of IAV NPs shows distinct differences from those of VSV, rabie virus, etc. The tail loop enables the nucleoprotein to form a homo-trimer with the RNA-binding groove exposed to the exterior of the nucleoprotein oligomer, suggesting that the bound RNA cannot be well protected by IAV NP (Ye et al., 2006; Ng et al., 2008).

Although IAV NP shows strong binding affinity with RNA, these bound RNA could be easily degraded by exogenous nuclease. A recent work reported the crystal structure of NP from influenza B virus (IBV) (PDB code: 3TJ0) ( $\mathrm{Ng}$ et al., 2012). Although NPs from IAV and IBV show high primary

B
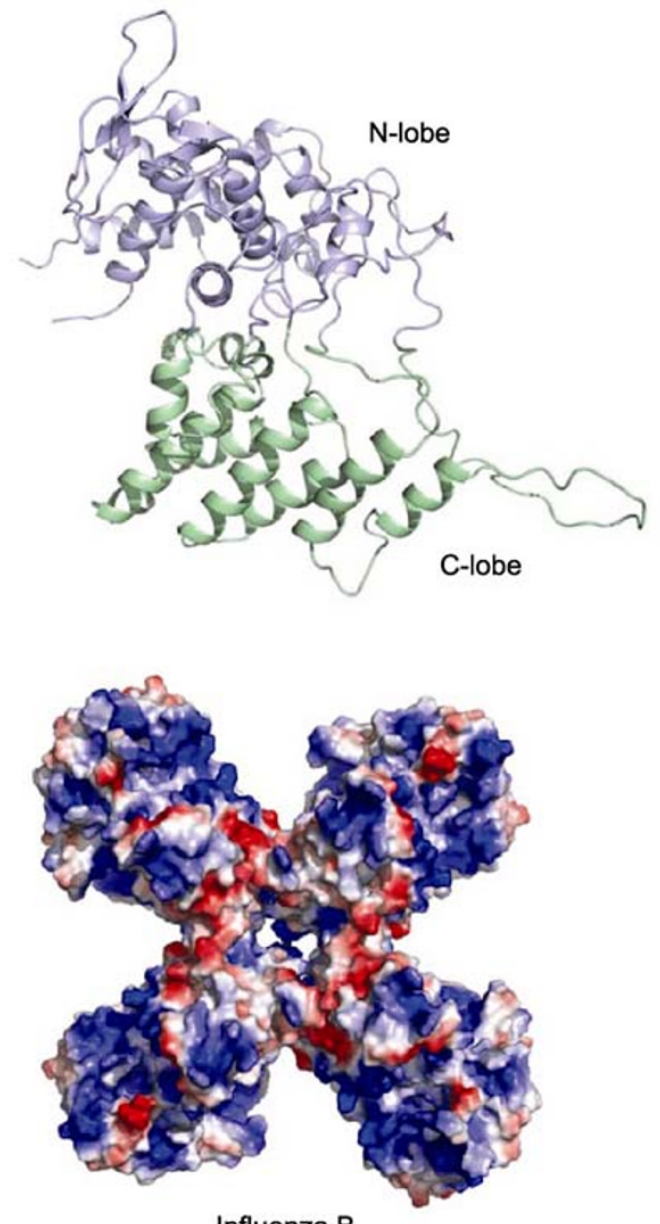

Influenza B

Figure 3. Comparison of nucleocapsid proteins of influenza A virus and influenza $B$ virus. Crystal structures of NP complex from IAV (PDB code: $2 \mathrm{IQH}$ and 2Q06) (A) and IBV (PDB code: 3TJ0) (B) are shown in the same orientation in upper panels. The $\mathrm{N}$-lobe and C-lobe for each NP-protomer are colored as blue and green, respectively. The homo-trimer and homo-tetramer of IAV and IBV NP are covered with potential surface in the bottom panels. 
sequence and structural similarities with few conformational shifts in the extended portions, their oligomerization states are totally different. IBV NP presents a homo-tetramer instead of the homo-trimer in IAV NP, suggesting the structural reorganization of the helix-loop-helix motif and the maintenance of some conserved hydrophobic contacts in higher-order NP oligomer formation ( $\mathrm{Ng}$ et al., 2012). The controversies surrounding the oligomerization of NP in influenza virus RNP can be settled by further performing detailed structural studies on a real RNP instead of NP alone.

\section{THE BIOLOGICAL ROLES OF LASSA FEVER VIRUS NP}

Structural research on Lassa fever virus (LASV) provided the evidence needed to extend the understanding of virally encoded NPs beyond RNA encapsidation. LASV belongs to the Arenaviridae family, which causes severe viral hemorrhagic fevers in humans with high morbidity and mortality; no vaccine has been developed for the virus thus far and only limited treatment is available to address LASV (Khan et al., 2008). LASV is a -ssRNA virus with two genomic RNA segments encoding four genes (Buchmeier et al., 2007). Nucleoprotein plays essential roles in LASV replication/transcription and host immune suppression (Martinez-Sobrido et al., 2007).

Qi and colleagues (Qi et al., 2011) presented the fulllength LASV NP structure (PDB code: $3 M X 2$ ), revealing two distinct $\mathrm{N}$ - and $\mathrm{C}$-terminal domains unlike other reported viral NPs (Fig. 4A). The C-terminal domain of LASV NP is highly similar in structure to the $3^{\prime}-5^{\prime}$ exonuclease/exoribonuclease superfamily. Further analysis confirms that LASV NP shows metal-dependent exoribonuclease activity and demonstrates

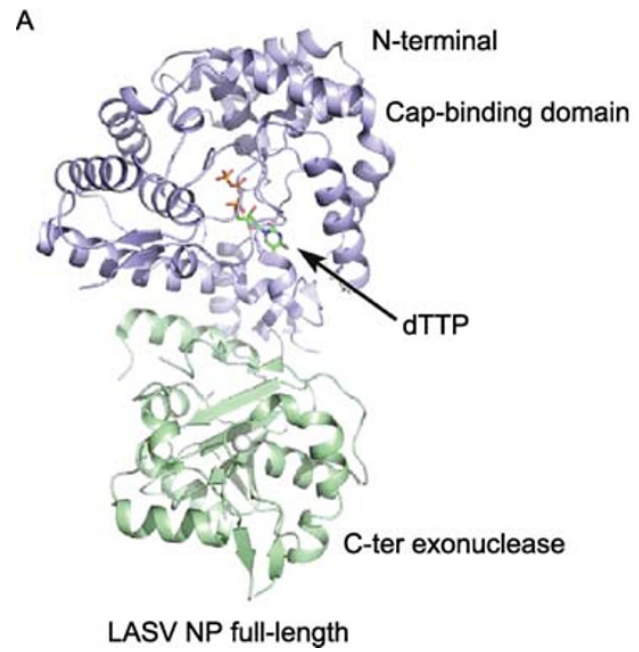

that this activity is responsible for host immune suppression. This is the first time to observe an enzymatic activity in a virally encoded NP. Based on the complex structure of LASV NP with dTTP (a known RNA cap analog), the N-terminal domain of LASV NP appears to act as a cap-binding factor. Thus, the author concluded that NP directly contributes to the cap-snatching process of viral replication.

Another independent group published their structural findings on LASV NP in two successive papers. In the first paper, the group confirmed the ssRNA-specific 3'-5' exoribonuclease activity and located this activity in the C-terminal domain of LAFV NP (PDB code: 3Q7B) (Hastie et al., 2011a). However, a second study showed significant variations in the biological role of the N-terminal domain of LAFV NP (Hastie et al., 2011b). Using a C-terminal truncated construct (1-340), they presented the complex structure of the $\mathrm{N}$-terminal domain of LAFV NP in complex with ssRNA (PDB code: 3T5Q) and designated this domain to be an RNA-binding domain. This domain controls RNA binding through a gating mechanism using $\alpha 5$ and $a 6$ (Fig. 4B). The results of this study notably revealed that the N-terminal domain of LAFV NP is unlikely to bind with cap or its analogs in vitro (Hastie et al., 2011b).

The inconsistencies on the biological functions of the $\mathrm{N}$-terminal domain of LAFV NP may be ascribed to their different constructions. For example, Qi et al. (2011) used full-length protein to examine cap-binding ability whereas Hastie et al. (2011b) used the truncation form without a C-terminal exonuclease domain to show the RNA binding feature. Although the precise biological function of the $\mathrm{N}$-terminal domain of LAFV NP remains controversial, these studies nevertheless are the first to discover the enzymatic activity of virally encoded NP and extend our understanding of the biological roles of NP in the viral life cycle.

B

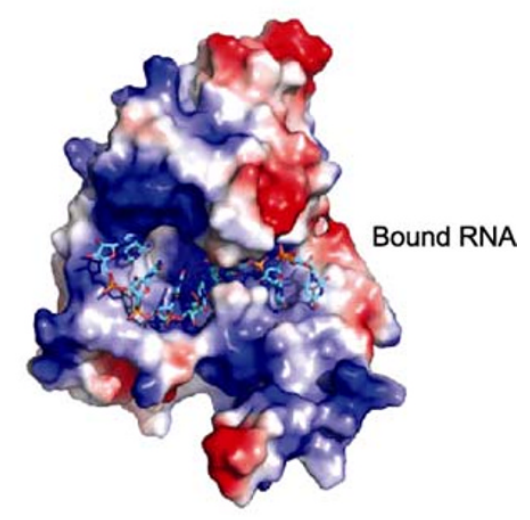

LASV NP N-domain-RNA

Figure 4. Structural information of LASV NP. (A) Crystal structure of full-length LASV NP (PDB code: 3MX2) is shown as colored cartoon diagram, in which the N-terminal cap-binding domain and C-terminal exonuclease domain are colored as blue and green, respectively. The bound dTTP, which is considered to be a cap analog, is presented as a colored stick. (B) The crystal structure of the N-terminal domain of LASV NP in complex with RNA (PDB code: 3T5Q). The polypeptide of the N-terminal domain of LASV NP is covered with potential surface, while the bound RNA molecule is shown as colored sticks. 


\section{THE VARIABLE FUNCTIONS OF BUNYAVIRUS NPS}

The Bunyaviridae family is the largest family of NSVs and comprises over 350 members that cause severe infectious diseases throughout animals, insects, and plants. The Bunyaviridae family consists of six major genera, i.e., Hantavirus (type species: Hantaan virus), Nairovirus (type species: Crimean-Congo hemorrhagic fever virus), Orthobunyavirus (type species: Bunyamwera virus), Phlebovirus (type species: Rift Valley fever virus), Tospovirus (type species: Tomato spotted wilt virus), and Uukuvirus (type species: Uukuniemi virus). All Bunyaviridae members have typical tripartite genomes ( $L, M$, and $S$ segments) that are encapsidated in the form of individual ribonucleoprotein complexes, preliminarily encoding four structural proteins, namely, the viral polymerase L, the glycoproteins Gc and Gn, and the NP. Some bunyaviruses also use an ambi-sense strategy, encoding the nonstructural proteins NSs and NSm (Guu et al., 2012). According to previous studies on the function of nucleoproteins in bunyaviruses, some NPs may be able to recognize specific viral RNA sequences (Osborne and Elliott, 2000; Mir et al., 2006; Ogg and Patterson, 2007) but most of the NPs bind to single-stranded RNA in a non-specific manner (Gott et al., 1993; Mohl and Barr, 2009). Although all Bunyaviridae members share high genomic similarity and similar replication/transcription features, their NPs show significant difference, either in terms of molecular weight or biological functions. The NP of Crimean-Congo hemorrhagic fever virus (CCHFV) from Nairovirus has the largest molecular weight (52 kDa). Hantaan virus from Hantavirus has a smaller NP with a molecular weight of $40 \mathrm{kDa}$; the molecular weights of NPs from the other four genera range from $25 \mathrm{kDa}$ to $31 \mathrm{kDa}$. The Bunyaviridae family includes hundreds of different genera and the nucleoproteins of each genus show little homology or other features in common; thus, elucidating the exact function and mechanism of each group of nucleoproteins case-by-case is necessary to understand bunyavirus replication and assembly.

The first structural study on Bunyaviridae members that encode NPs focused on the Rift Valley fever virus (RVFV) nucleoprotein. RVFV is the causative agent of Rift Valley fever in livestock and humans, which is endemic to sub-Saharan Africa, and belongs to the Phlebovirus genus. The first crystal structure of RVFV NP was solved through a denaturation/renaturation method to obtain the RNA-free NP protein (PDB code: 3LYF) (Raymond et al., 2010) (Fig. 5A). RVFV NP has a novel helical fold that is compact and well-ordered at both the $\mathrm{N}$ and $\mathrm{C}$ termini. However, this structure shows no positively charged surface cleft for RNA binding and no protruding termini or loops to stabilize a defined N-RNA oligomer or RNP helix like NPs from other negative-sense RNA viruses.

Using a novel purification method, another group solved the crystal structure of RVFV NP in the native form (PDB code: 3OU9) (Ferron et al., 2011). In this structure, RVFV NP presents a hexameric ring-shaped form to hold a distinct functional RNA binding site (Fig. 5B). Although the parts of these two structures show high identity, significant conformational difference occurs at the N-terminal arm of RVFV NP, which is composed of the first 30 amino acids (Fig. 5C). In the monomeric structure, the $\mathrm{N}$-terminal arm interacts with the body part of the same molecule, forms a compact architecture, and shadows the functional RNA binding groove in the monomeric structure. In sharp contrast to the hexameric structure, the N-terminal arm extends and interacts with adjacent molecules to form a ring-shaped oligomer, and the RNA binding site is exposed to the solvent. These two distinct positions of the $\mathrm{N}$-terminal arm may reflect the structural change that has to occur for the formation of RNP.

Following the structural research on RVFV NP, the crystal structure of CCHFV NP (strain YL04057), the largest Bunyaviridae member that encodes NP with a molecular weight of $52 \mathrm{kDa}$, revealed an unexpected biological function (Guo et al., 2012). Unlike the RVFV NP, the full-length CCHFV NP protein mainly exists in a monomeric form after DNase/ RNase treatment. CCHFV NP possesses a racket- shaped overall structure and features two major parts: a "head" domain (M1-I180 and A300-1482) and a "stalk" domain (R181-A299) (Fig. 6A). Both head and stalk domains are predominantly composed of $\alpha$-helices. There is a large positively charged cavity located at the center of the head domain and a positively charged region in the stalk domain adjacent to the head domain (Guo et al., 2012). Although CCHFV NP has no primary sequence homologs, its head domain shows high structural similarity with the LASV NP N-terminal domain (Fig. 6B). However, CCHFV NP (or the head domain alone) shows functions distinctly different from the $\mathrm{N}$-terminal domain of LASV NP. CCHFV NP displays very low binding affinity with either cap/cap analog or exogenous RNA, suggesting that it (or, at least, its monomeric form) is unlikely to possess a cap-snatching or RNA-binding function. CCHFV NP has intrinsic nuclease activities on both singleand double-stranded DNA that may be stimulated to different extents by divalent cations, such as $\mathrm{Mn}^{2+}, \mathrm{Co}^{2+}$, and $\mathrm{Mg}^{2+}$ (Fig. 6C). Two independent groups have published their work on the CCHFV NP structure (Carter et al., 2012; Wang et al., 2012). The structure of NP from CCHFV strain Baghdad-12 shows head and stalk domains with highly similar structures. However, the orientation of the stalk domain from Baghdad-12 strain shows a significant difference from the previous one. This structural flexibility may suggest the basis for NP conformation switching during the RNA-binding process. Based on structural information on CCHFV NP (strain IbAr10200) in two distinct forms, Wang and colleagues (Wang et al., 2012) proposed a conformational change process for nucleoprotein oligomerization. All of these results provide new insights into the biological role of NPs in Bunyavirus replication. 
A

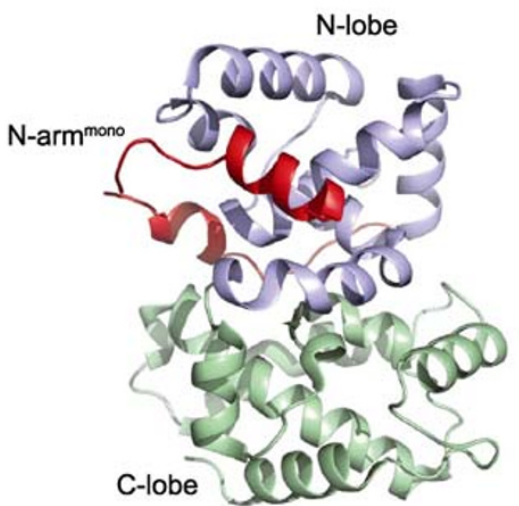

RVFV monomeric NP
B

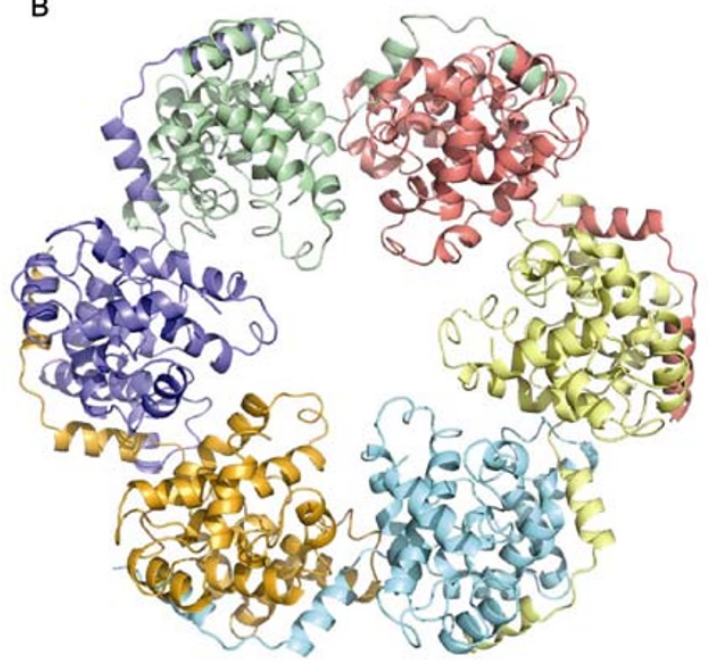

RVFV hexameric NP ring

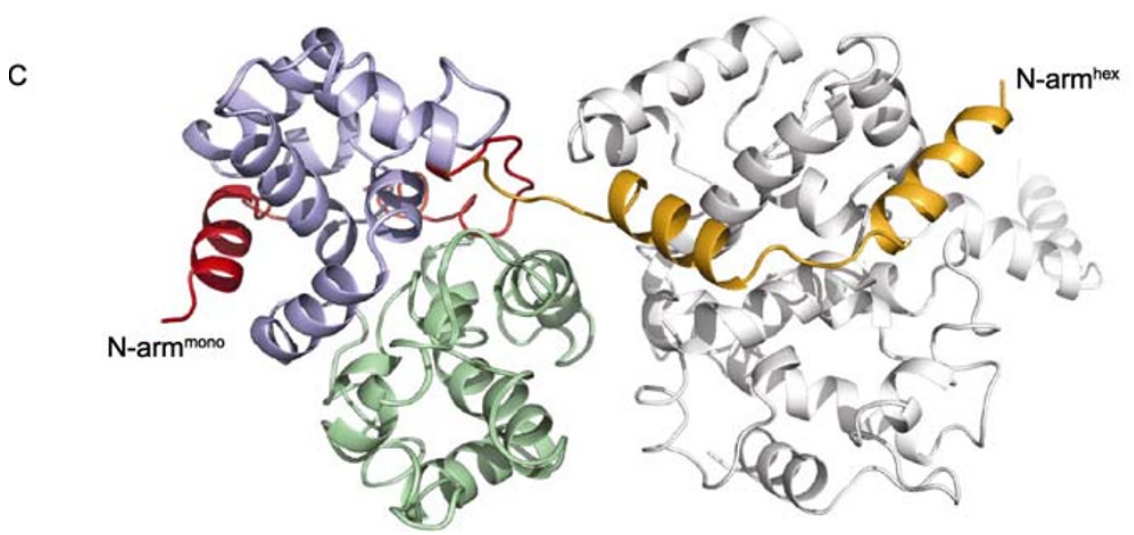

Figure 5. Crystal structures of RVFV NP in different oligomerization state. (A) Crystal structures of the monomeric RVFV NP achieved through dnature/renature methods (PDB code: 3 LYF). The $\mathrm{N}$-arm, N-lobe and C-lobe are colored as red, blue and green, respectively. (B) Crystal structure of the ring-shaped hexameric RVFV NP (PDB code: 3OU9). (C) Structural comparison between the monomeric and oligomeric RVFV NP. An adjacent molecule in the hexameric NP ring is shown as white cartoon to indicated the position of $\mathrm{N}$-arm in oligomerization state. The $\mathrm{N}$-lobe and C-lobe could be perfectly superimposed, while the $\mathrm{N}$-arm presented distinct conformational change. The $\mathrm{N}$-arms in monomeric state and hexameric state are colored with red and orange, respectively.

\section{PERSPECTIVES}

Current structural and functional studies on -sSRNA viruses that encode NPs provide valuable information that boost our understanding of how these NPs function in the viral life cycle as structural blocks or sites of enzymatic activities. Viral NP is one of the most important components in the formation of -ssRNA virus RNPs and, thus, viral replication/transcription. Therefore, it is conceivable that reagents that can inhibit either RNA encapsidation activity or the enzymatic activity of viral NP could potentially prohibit viral replication. This hypothesis was first approved in IAV NP (Kao et al., 2010). Using forward chemical genetics, a chemical compound, nucleozin, was discovered to trigger the aggregation of IAV NP, thus inhibiting nuclear accumulation and virus replication with a nanomolar median effective concentration. As antiviral resistance to available drugs, such as adamantanes or neuraminidase inhibitors, has rapidly emerged, new insights into the development of new antiviral targets and new drugs for influenza virus infections have been gained.

Current structural investigations have revealed the structural details and molecular mechanism of -ssRNA virus NPs and provided plausible ways to prohibit viral replication acting on viral NPs. However, some questions remain. For example, what is the "real" oligomerization state of viral NP in the RNP? What dynamic mechanism is used by NPs to form the highly 
A

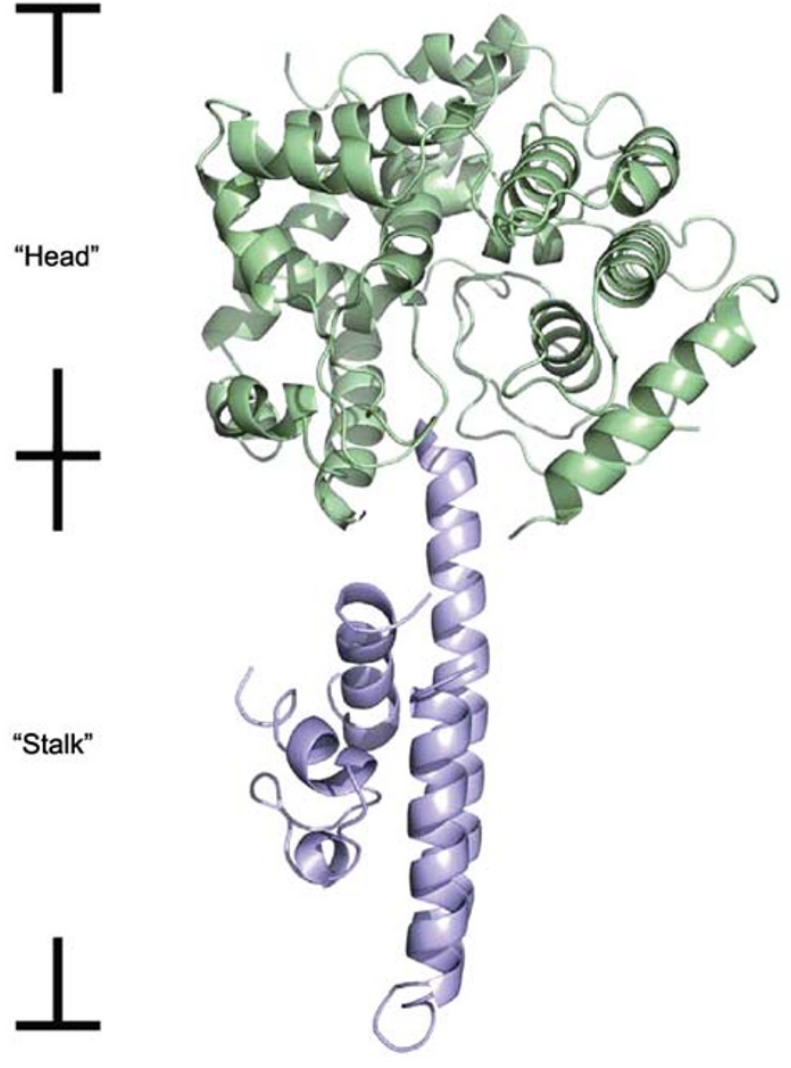

B

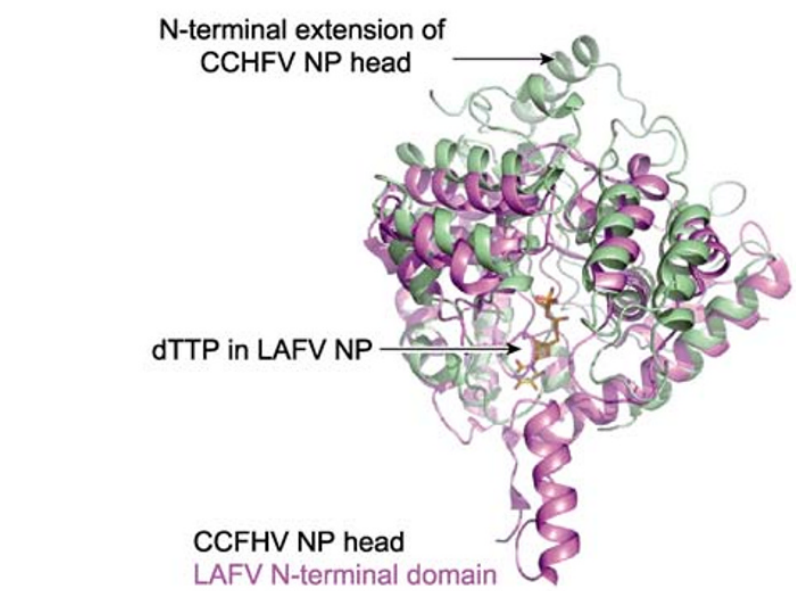

C

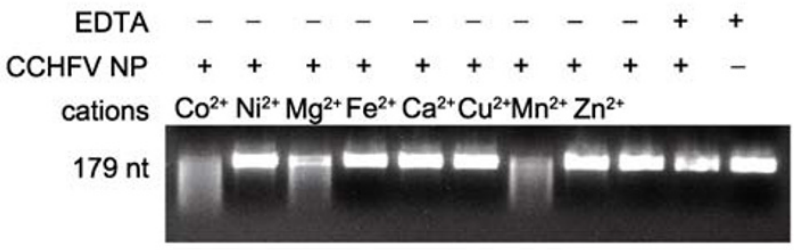

dsDNA, $37^{\circ} \mathrm{C}, 15 \mathrm{~min}, \mathrm{pH} 7.0$

Figure 6. Crystal structure of CCHFV NP (strain YL04057). (A) Crystal structure of full-length CCHFV NP from strain YL04057 (PDB code: $3 \mathrm{U} 3 \mathrm{I}$ ) is shown as a cartoon diagram. The head domain and stalk domain are colored as green and blue, respectively. (B) The structural comparison of head domain of CCHFV NP and N-terminal domain of LASV. The head domain of CCHFV NP and $\mathrm{N}$-terminal domain of LASV are colored as green and magenta, respectively. The bound dTTP in LASV NP is shown as a colored stick. (C) The endonuclease activity of CCHFV NP is enhanced by cations. (During the preparation of this manuscript, the coordinate file of CCHFV (strain Baghdad-12) is not yet released in PDBank and we, therefore, do not show the structural comparison between two strains.)

ordered structures of RNPs? Is there any consistency or variation in the structure or function of NPs from the same virus family? All of these questions require further structural studies on -ssRNA viruses that encode NPs or RNPs.

\section{ACKNOWLEDGMENTS}

This work was supported by the National Natural Science Foundation of China (Grant Nos. 31000332 and 31170678) and the Fundamental Research Funds for the Central Universities.

\section{ABBREVIATIONS}

-ssRNA, negative-sense single-stranded RNA; BDV, borna disease virus; CCHFV, Crimean-Congo hemorrhagic fever virus; IAV, influenza A virus; IBV, influenza B virus; LASV, Lassa fever virus; NP, nucleocapsid protein; RdRp, RNA-dependent RNA polymerase; RSV, respiratory syncytial virus; RVFV, Rift Valley fever virus; VSV, ve- sicular stomatitis virus

\section{REFERENCES}

Albertini, A.A., Schoehn, G., Weissenhorn, W., and Ruigrok, R.W. (2008). Structural aspects of rabies virus replication. Cell Mol Life Sci 65, 282-294.

Albertini, A.A., Wernimont, A.K., Muziol, T., Ravelli, R.B., Clapier, C.R., Schoehn, G., Weissenhorn, W., and Ruigrok, R.W. (2006). Crystal structure of the rabies virus nucleoprotein-RNA complex. Science 313, 360-363.

Area, E., Martin-Benito, J., Gastaminza, P., Torreira, E., Valpuesta, J.M., Carrascosa, J.L., and Ortin, J. (2004). 3D structure of the influenza virus polymerase complex: localization of subunit domains. Proc Natl Acad Sci U S A 101, 308-313.

Buchmeier M.J., Peters, C.J., and de la Torre, J.C. (2007). Arenaviridae: The viruses and their replication. Fields Virology eds Knipe, D.M., Howley, P.M. (Lippincott Williams \& Wilkins,Philadelphia), 5th Ed, 1791-1827. 
Carter, S.D., Surtees, R., Walter, C.T., Ariza, A., Bergeron, E., Nichol, S.T., Hiscox, J.A., Edwards, T.A., and Barr, J.N. (2012). Structure, function, and evolution of the crimean-congo hemorrhagic Fever virus nucleocapsid protein. J Virol 86, 10914-10923.

Emerson, S.U., and Schubert, M. (1987). Location of the binding domains for the RNA polymerase $L$ and the ribonucleocapsid template within different halves of the NS phosphoprotein of vesicular stomatitis virus. Proc Natl Acad Sci U S A 84, 5655-5659.

Ferron, F., Li, Z., Danek, E.I., Luo, D., Wong, Y., Coutard, B., Lantez, V., Charrel, R., Canard, B., Walz, T., et al. (2011). The hexamer structure of Rift Valley fever virus nucleoprotein suggests a mechanism for its assembly into ribonucleoprotein complexes. PLoS Pathog 7, e1002030.

Ge, P., Tsao, J., Schein, S., Green, T.J., Luo, M., and Zhou, Z.H. (2010). Cryo-EM model of the bullet-shaped vesicular stomatitis virus. Science 327, 689-693.

Gött, P., Stohwasser, R., Schnitzler, P., Darai, G., and Bautz E.K. (1993). RNA binding of recombinant nucleocapsid proteins of hantaviruses. Virology 194, 332-337.

Green, T.J., and Luo, M. (2009). Structure of the vesicular stomatitis virus nucleocapsid in complex with the nucleocapsid-binding domain of the small polymerase cofactor. Proc Natl Acad Sci U S A 106, 11713-11718.

Green, T.J., Rowse, M., Tsao, J., Kang, J., Ge, P., Zhou, Z.H., and Luo, M. (2011). Access to RNA encapsidated in the nucleocapsid of vesicular stomatitis virus. J Virol 85, 2714-2722.

Green, T.J., Zhang, X., Wertz, G.W., and Luo, M. (2006). Structure of the vesicular stomatitis virus nucleoprotein-RNA complex. Science 313, 357-360.

Guo, Y., Wang, W., Ji, W., Deng, M., Sun, Y., Zhou, H., Yang, C., Deng, F., Wang, H., Hu, Z., et al. (2012). Crimean-Congo hemorrhagic fever virus nucleoprotein reveals endonuclease activity in bunyaviruses. Proc Natl Acad Sci U S A 109, 5046-5051.

Guu, T.S., Zheng, W., and Tao, Y.J. (2012). Bunyavirus: structure and replication. Adv Exp Med Biol 726, 245-266.

Hastie, K.M., Kimberlin, C.R., Zandonatti, M.A., MacRae, I.J., and Saphire, E.O. (2011a). Structure of the Lassa virus nucleoprotein reveals a dsRNA-specific $3^{\prime}$ to $5^{\prime}$ exonuclease activity essential for immune suppression. Proc Natl Acad Sci U S A 108, 2396-2401.

Hastie, K.M., Liu, T., Li, S., King, L.B., Ngo, N., Zandonatti, M.A., Woods, V.L., Jr., de la Torre, J.C., and Saphire, E.O. (2011b). Crystal structure of the Lassa virus nucleoprotein-RNA complex reveals a gating mechanism for RNA binding. Proc Natl Acad Sci U S A 108, 19365-19370.

Iseni, F., Barge, A., Baudin, F., Blondel, D., and Ruigrok, R.W. (1998). Characterization of rabies virus nucleocapsids and recombinant nucleocapsid-like structures. J Gen Virol 79 (Pt 12), 2909-2919.

Kao, R.Y., Yang, D., Lau, L.S., Tsui, W.H., Hu, L., Dai, J., Chan, M.P., Chan, C.M., Wang, P., Zheng, B.J., et al. (2010). Identification of influenza A nucleoprotein as an antiviral target. Nat Biotechnol 28, 600-605.

Khan, S.H., Goba, A., Chu, M., Roth, C., Healing, T., Marx, A., Fair, J., Guttieri, M.C., Ferro, P., Imes, T., et al. (2008). New opportunities for field research on the pathogenesis and treatment of Lassa fever. Antiviral Res 78, 103-115.

Kranzusch, P., and Whelan, S. (2012). Architecture and regulation of negative-strand viral enzymatic machinery. RNA Biol 9.
Lansing, M.P., John, P.H., and Donald, A.K. (1998). Microbiology, 4 edn (William C Brown Pub).

Martinez-Sobrido, L., Giannakas, P., Cubitt, B., Garcia-Sastre, A., and de la Torre, J.C. (2007). Differential inhibition of type I interferon induction by arenavirus nucleoproteins. J Virol 81, 12696-12703.

Mir, M.A., Brown, B., Hjelle, B., Duran, W.A., and Panganiban, A.T. (2006). Hantavirus N protein exhibits genus-specific recognition of the viral RNA panhandle. J Virol 80, 11283-11292.

Mohl B.P., and Barr, J,N. (2009). Investigating the specificity and stoichiometry of RNA binding by the nucleocapsid protein of Bunyamwera virus. RNA 15, 391-399.

Ng, A.K., Lam, M.K., Zhang, H., Liu, J., Au, S.W., Chan, P.K., Wang, J., and Shaw, P.C. (2012). Structural basis for RNA binding and homo-oligomer formation by influenza B virus nucleoprotein. J Virol 86, 6758-6767.

Ng, A.K., Zhang, H., Tan, K., Li, Z., Liu, J.H., Chan, P.K., Li, S.M., Chan, W.Y., Au, S.W., Joachimiak, A., et al. (2008). Structure of the influenza virus A H5N1 nucleoprotein: implications for RNA binding, oligomerization, and vaccine design. Faseb $\mathrm{J} 22$, 3638-3647.

Ogg M.M., and Patterson J.L. (2007). RNA binding domain of Jamestown Canyon virus $S$ segment RNAs. J Virol 81, 13754-13760.

Osborne J.C., and Elliott R.M. (2000). RNA binding properties of bunyamwera virus nucleocapsid protein and selective binding to an element in the $5^{\prime}$ terminus of the negative-sense $\mathrm{S}$ segment. $\mathrm{J}$ Virol 74, 9946-9952.

Qi, X., Lan, S., Wang, W., Schelde, L.M., Dong, H., Wallat, G.D., Ly, H., Liang, Y., and Dong, C. (2011). Cap binding and immune evasion revealed by Lassa nucleoprotein structure. Nature 468, 779-783.

Raymond, D.D., Piper, M.E., Gerrard, S.R., and Smith, J.L. (2010). Structure of the Rift Valley fever virus nucleocapsid protein reveals another architecture for RNA encapsidation. Proc Natl Acad Sci U S A 107, 11769-11774.

Rudolph, M.G., Kraus, I., Dickmanns, A., Eickmann, M., Garten, W., and Ficner, R. (2003). Crystal structure of the borna disease virus nucleoprotein. Structure 11, 1219-1226.

Ruigrok, R.W., Crepin, T., and Kolakofsky, D. (2011). Nucleoproteins and nucleocapsids of negative-strand RNA viruses. Curr Opin Microbiol 14, 504-510.

Tawar, R.G., Duquerroy, S., Vonrhein, C., Varela, P.F., Damier-Piolle, L., Castagne, N., MacLellan, K., Bedouelle, H., Bricogne, G., Bhella, D., et al. (2009). Crystal structure of a nucleocapsid-like nucleoprotein-RNA complex of respiratory syncytial virus. Science 326, 1279-1283.

Thomas, D., Newcomb, W.W., Brown, J.C., Wall, J.S., Hainfeld, J.F., Trus, B.L., and Steven, A.C. (1985). Mass and molecular composition of vesicular stomatitis virus: a scanning transmission electron microscopy analysis. J Virol 54, 598-607.

Wang, Y., Dutta, S., Karlberg, H., Devignot, S., Weber, F., Hao, Q., Tan, Y.J., Mirazimi, A., and Kotaka, M. (2012). Structure of Crimean-Congo Haemorraghic Fever Virus Nucleoprotein: Superhelical Homo-Oligomers and the Role of Caspase-3 Cleavage. J Virol.

Xue, F., Sun, Y., Yan, L., Zhao, C., Chen, J., Bartlam, M., Li, X., Lou, 
Z., and Rao, Z. (2010). The crystal structure of porcine reproductive and respiratory syndrome virus nonstructural protein Nsp1beta reveals a novel metal-dependent nuclease. J Virol 84, 6461-6471.

Ye, Q., Krug, R.M., and Tao, Y.J. (2006). The mechanism by which influenza A virus nucleoprotein forms oligomers and binds RNA. Nature 444, 1078-1082.

Yuan, P., Bartlam, M., Lou, Z., Chen, S., Zhou, J., He, X., Lv, Z., Ge,
R., Li, X., Deng, T., et al. (2009). Crystal structure of an avian influenza polymerase $\mathrm{PA}(\mathrm{N})$ reveals an endonuclease active site. Nature 458, 909-913.

Zhao, C., Lou, Z., Guo, Y., Ma, M., Chen, Y., Liang, S., Zhang, L., Chen, S., Li, X., Liu, Y., et al. (2009). Nucleoside monophosphate complex structures of the endonuclease domain from the influenza virus polymerase PA subunit reveal the substrate binding site inside the catalytic center. J Virol 83, 9024-9030. 